\title{
MIXED NORM $n$-WIDTHS
}

\author{
C. DE BOOR ${ }^{1}$, R. DEVORE ${ }^{2}$ AND K. HÖLLIG ${ }^{3}$
}

\begin{abstract}
Recently, the Soviet mathematicians R. Ismagilov [4], E. Gluskin [3] and B. Kashin [5] have obtained some deep and surprising results on $n$-widths for Sobolev spaces in the mixed norm case. In this note, we will give a new and simpler proof of Gluskin's result and show its connection with a certain classical combinatorial problem.
\end{abstract}

0. Introduction. Let $X_{p}=L_{p}[0,1], 1<p<\infty$, and $X_{\infty}=C[0,1]$. We denote by $W_{p}^{k}$ the Sobolev space of all $f$ with $f^{(k-1)}$ absolutely continuous and $f^{(k)} \in X_{p}$ equipped with the norm $\|f\|_{p, k}:=\|f\|_{p}+\left\|f^{(k)}\right\|_{p}$. The unit ball $U\left(W_{p}^{k}\right)$ is a compact subset of $X_{q}$ in case $k>1 / p-1 / q$ and so the $n$-width

$$
d_{n}\left(U\left(W_{p}^{k}\right)\right)_{q}:=\inf _{\operatorname{dim}(Y)=n} \sup _{f \in U\left(W_{p}^{k}\right)} \inf _{g \in Y}\|f-g\|_{q}
$$

tends to zero as $n \rightarrow \infty$. We are interested in characterizing the asymptotic behavior of $d_{n}$ and finding asymptotically optimal subspaces $Y_{n}$.

When $q=p$, this is quite straightforward as $d_{n}\left(U\left(W_{p}^{k}\right)\right)_{p} \sim n^{-k}$ and either of the two spaces, polynomials of degree $<n$ or smooth splines of order $k$ with $n-k$ equally spaced knots, is asymptotically optimal. When $q \neq p$, the so-called mixed norm case, the problem becomes more substantial for certain values of $p$ and $q$, and a solution for the complete range $1<p, q<\infty$ was only recently given by Kashin [5]. One of the early breakthroughs was due to Gluskin [3] who showed that

$$
d_{n}\left(U\left(W_{1}^{2}\right)\right)_{\infty}<\text { const } n^{-3 / 2} \text {. }
$$

This result is quite surprising in view of the fact that the best possible embedding is $W_{1}^{2} \subseteq W_{\infty}^{1}$ from which one suspects $d_{n}\left(U\left(W_{1}^{2}\right)\right) \sim n^{-1}$. The lower estimate $d_{n}\left(U\left(W_{1}^{2}\right)\right)_{\infty}>$ const $n^{-3 / 2}$ is an immediate consequence of the estimate $d_{n}\left(U\left(W_{1}^{2}\right)\right)_{2} \sim n^{-3 / 2}$ which is an old result of Stechkin [7] based on a clever argument of W. Rudin, so (0.1) actually determines the $n$-width of $U\left(W_{1}^{2}\right)$, i.e. $d_{n}\left(U\left(W_{1}^{2}\right)\right)_{\infty} \sim n^{-3 / 2}$.

Gluskin's proof of $(0.1)$ follows the ideas of Ismagilov [4] who had given an earlier estimate $d_{n}\left(U\left(W_{1}^{2}\right)\right)_{\infty}<$ const $n^{-6 / 5}$. Their technique is to reduce the problem to the study of $n$-widths of the unit ball of $l_{1}^{m}$ in the space $l_{\infty}^{m}$. This approach

Received by the editors March 7, 1979.

AMS (MOS) subject classifications (1970). Primary 41A45; Secondary 41A15, 41A25.

Key words and phrases. Mixed norm $n$-widths, Sobolev spaces, combinatorics.

'Supported by the U. S. Army under Contract No. DAAG 29-75-C-0024.

${ }^{2}$ Supported by NSF Grant MCS 77-22982.

${ }^{3}$ This research was initiated at the Universität Bonn under the support of Sonderforschungsbereich 72. 
offers little insight into the nature of asymptotically optimal subspaces.

In $\$ 2$, we will give a new proof of $(0.1)$ by explicit construction of an asymptotically optimal subspace. This construction is based on the solution of a combinatorial problem which, as we realized later, has many equivalent formulations such as the existence of orthogonal Latin squares or more general block designs or of finite projective planes. Its solution, in \$3, uses the existence of finite fields of prime power order. Our construction also has the advantage of being linear and so we not only determine $d_{n}$ asymptotically but the linear $n$-width as well.

1. Proof outline. The idea of our proof is (i) to replace $f \in U\left(W_{1}^{2}\right)$ by its broken line interpolant $S$ on a uniform mesh of width $n^{-3 / 2}$ (as is already done in Gluskin [3]), at an error of no more than $\left\|f^{\prime \prime}\right\|_{1} n^{-3 / 2}$, (ii) to observe that $S=f(0)+$ $\sum_{i} \alpha_{i}\left(\cdot-x_{i}\right)_{+}$with $\Sigma_{i}\left|\alpha_{i}\right|<2\left\|f^{\prime \prime}\right\|_{1}$, and (iii) to construct $2 n+1$ broken lines (or, linear splines) on the same mesh whose span is within $n^{-3 / 2}$ of each of the functions $\left(\cdot-x_{i}\right)_{+}, i=0, \ldots, n^{3 / 2}$.

The first two steps are covered by the following well-known proposition.

Proposition 1. If $f \in W_{1}^{2}$ and $S$ is the piecewise linear spline with knots $x_{i}=i / N, i=0, \ldots, N$, which interpolates $f$ at each $x_{i}$, then

$$
\|f-S\|_{\infty}<N^{-1}\left\|f^{\prime \prime}\right\|_{1}
$$

and

$$
S(x)=f(0)+\sum_{i=0}^{N-1} \alpha_{i}\left(x-x_{i}\right)_{+} \text {with } \sum_{i=1}^{N-1}\left|\alpha_{i}\right|<2\left\|f^{\prime \prime}\right\|_{1} \text {. }
$$

Proof. This is well known but we give the simple proof. Since $S^{\prime}(x)=$

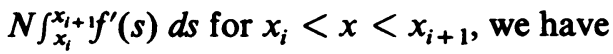

$$
\begin{aligned}
|f(x)-S(x)| & =\left|\int_{x_{i}}^{x}\left(f^{\prime}(t)-S^{\prime}(t)\right) d t\right|=N\left|\int_{x_{i}}^{x} \int_{x_{i}}^{x_{i+1}}\left[f^{\prime}(t)-f^{\prime}(s)\right] d s d t\right| \\
& <N \int_{x_{i}}^{x_{i+1}} \int_{x_{i}}^{x_{i+1}} \int_{x_{i}}^{x_{i+1}}\left|f^{\prime \prime}(r)\right| d r d s d t<N^{-1}\left\|f^{\prime \prime}\right\|_{1}
\end{aligned}
$$

which is (1.1).

If now $S=f(0)+\sum_{i=0}^{N-1} \alpha_{i}\left(\cdot-x_{i}\right)_{+}$, then $\alpha_{i}=S_{i}^{\prime}-S_{i-1}^{\prime}$ with $S_{i}^{\prime}:=N \int_{x_{1}}^{x_{1}+1 f^{\prime}}(s) d s$ for $i>0$ and $S_{-1}^{\prime}:=0$. Thus, for $0<i<n$,

$$
\begin{aligned}
\left|\alpha_{i}\right| & =N\left|\int_{x_{i}}^{x_{i+1}}\left[f^{\prime}(t)-f^{\prime}\left(t-N^{-1}\right)\right] d t\right| \\
& <N \int_{x_{i}}^{x_{i+1}} \int_{x_{i-1}}^{x_{i+1}}\left|f^{\prime \prime}(s) d s\right| d t=\int_{x_{i-1}}^{x_{i+1}}\left|f^{\prime \prime}(r)\right| d r
\end{aligned}
$$

from which (1.2) follows.

2. Construction of an asymptotically optimal subspace. We consider $\boldsymbol{n}$ of the form $n=m^{2}$ with $m>1$, and let $N=m^{3}$. Proposition 1 shows that the space $\mathfrak{S}_{N}$ of linear splines with the $N$ equally spaced knots $x_{i}=i / N, i=0, \ldots, N$, gives the correct order of approximation $N^{-1}=n^{-3 / 2}$ to $U\left(W_{1}^{2}\right)$ in $C$. However, this space has too high a dimension, namely $N+1$. We now construct within $\mathfrak{S}_{N}$ a space $Y_{n}$ 
of dimension $2 n+1$ which is within $O\left(N^{-1}\right)$ of $U\left(W_{1}^{2}\right)$.

Because of Proposition 1, it will be enough to construct $Y_{n}$ so that, for each $0<i<N$, there is a function $\Phi_{i} \in Y_{n}$ with

$$
\left\|\left(\cdot-x_{i}\right)_{+}-\Phi_{i}\right\|_{\infty}<\text { const } N^{-1}
$$

or, what is the same thing,

$$
\left|\int_{0}^{x}\left(t-x_{i}\right)_{+}^{0}-\phi_{i}(t) d t\right|<\text { const } N^{-1}
$$

where $\phi_{i}=\Phi_{i}^{\prime}$, and where const is independent of $N$ (and $x$ ). For this, we let $Y_{n}^{\prime}:=\left\{\Phi^{\prime}: \Phi \in Y_{n}\right\}$ and work to construct $Y_{n}^{\prime}$ of dimension $<2 n$ and containing, for each $i$, some $\phi_{i}$ for which (2.2) holds. The space $Y_{n}$ will then be recovered from $Y_{n}^{\prime}$ by integration and the adjoining of the constant functions.

We choose $Y_{n}^{\prime}$ as the linear span of $2 n$ step functions, each of the form

$$
\delta_{A_{i}}:=\sum_{\nu \in A_{i}} \delta_{\nu}
$$

with $A_{i}$ a subset of $\{1,2, \ldots, N\}$ of cardinality $m$, and

$$
\delta_{\nu}(t):=\left\{\begin{array}{ll}
1, & x_{\nu-1}<x<x_{\nu}, \\
0, & \text { otherwise, }
\end{array} \quad \nu=1, \ldots, N .\right.
$$

Specifically, we choose

$$
A_{n+i}:=\{(i-1) m+1, \ldots, i m\}, \quad i=1, \ldots, n,
$$

thus forming a very simple partition of $\{1, \ldots, N\}$. We also choose $A_{1}, \ldots, A_{n}$ as a partition of $\{1, \ldots, N\}$, but in a more complicated way to be made precise in the following.

Consider now one of the functions $\left(\cdot-x_{i}\right)_{+}^{0}$ which we would like to approximate with the accuracy of (2.2). If $(j-1) m<i<j m$, then

$$
\left(\cdot-x_{i}\right)_{+}^{0}=\sum_{\nu=i+1}^{N} \delta_{\nu}=\sum_{\nu=i+1}^{j m} \delta_{\nu}+\sum_{k=j+1}^{n} \delta_{A_{n+k}},
$$

leaving us with the problem of approximating the step function $\sum_{i+1}^{j m} \delta_{v}$. Now, we could approximate this function by $((j m-i) / m) \delta_{A_{n+j}}$. Then, with $e$ the error in this approximation, we would have

$$
\int_{0}^{x} e(t) d t=0 \quad \text { for } x \notin\left[x_{(j-1) m}, x_{j m}\right] \text {. }
$$

But, $\sup _{x}\left|\int_{0}^{x} e(t) d t\right|=((j m-i) / m)((i-(j-1) m) / N)$, and this can be as large as $m / 4 N$. So we must find a different approach and this is where the functions $\delta_{A}$, $i=1, \ldots, n$, enter the picture.

Since $A_{1}, \ldots, A_{n}$ is a partition for $\{1, \ldots, N\}$, there exists, for each $\nu \in$ $\{1, \ldots, N\}$, exactly one set $B_{\nu} \in\left\{A_{1}, \ldots, A_{n}\right\}$ for which $\nu \in B_{\nu}$. With this, the function $\sum_{v=i+1}^{j m} \delta_{B_{v}}$ in $Y_{n}^{\prime}$ provides a first approximation to $\Sigma_{i+1}^{j m} \delta_{\nu}$ whose error is of the form

$$
e_{i}:=-\sum_{\nu=i+1}^{j m} \sum_{\mu \in B_{\nu} \backslash\{\nu\}} \delta_{\mu}
$$


Again we can prevent this error from building up by subtracting off the mean value of this error on each interval $\left[x_{(k-1) m}, x_{k m}\right]=\operatorname{supp} \delta_{A_{n+k}}$. That is, for each $k=$ $1, \ldots, n$, let

$$
m_{i k}:=-N \int \delta_{A_{n+k}} e_{i}
$$

Then

$$
\phi_{i}:=\sum_{\nu=i+1}^{j m} \delta_{B_{\nu}}+\sum_{k=j+1}^{n} \delta_{A_{n+k}}-\sum_{k=1}^{n}\left(m_{i k} / m\right) \delta_{A_{n+k}}
$$

is an approximation to $\left(\cdot-x_{i}\right)_{+}^{0}$ from $Y_{n}^{\prime}$ for which

$$
\begin{aligned}
\int_{x_{(k-1) m}}^{x_{k m}}\left[\left(t-x_{i}\right)_{+}^{0}-\phi_{i}(t)\right] d t & =\int \delta_{A_{n+k}}(t)\left(e_{i}(t)+m_{i k} / m\right) d t \\
& =-m_{i k} / N+\left(m_{i k} / m\right)(m / N)=0, \quad k=1, \ldots
\end{aligned}
$$

Consequently, for $x_{(k-1) m} \leqslant x \leqslant x_{k m}$, we have

$$
\left|\int_{0}^{x}\left[\left(t-x_{i}\right)_{+}^{0}-\phi_{i}(t)\right] d t\right|=\left|\int_{x_{(k-1) m}}^{x}\left[e_{i}(t)+m_{i k} / m\right] d t\right|<m_{i k} / N .
$$

Thus, to obtain (2.2) with this construction, the second partition $A_{1}, \ldots, A_{n}$ for $\{1, \ldots, N\}$ must be chosen so that

$$
\max _{i, k} m_{i k}<\text { const independent of } n \text {. }
$$

Now we can, in fact, achieve $m_{i k}<1$ for all $i$ and $k$ if we manage to choose $A_{1}, \ldots, A_{n}$ so that

(2.6) $\operatorname{card}\left(A_{\nu} \cap A_{n+k}\right)<1$ for all $\nu, k$ and if both $A_{\nu}$ and $A_{\mu}$ intersect $A_{n+j}, \nu \neq \mu$, then, for all $k \neq j, A_{n+k}$ contains an integer from at most one of $A_{\nu}$ and $A_{\mu}$.

Indeed if (2.6) holds then the error function $e_{i}$ involves at most one $\delta_{\mu}$ with $\mu \in A_{n+k}$ for any $k$ and therefore $m_{i, k}<1$.

We do not know how to choose such a partition, in general. But, if $m$ is a prime power, then there is an easy such choice, and we give it in \$3. With this, we have

THEOREM 1. Let $n=m^{2}$ be any integer for which a partition $A_{1}, \ldots, A_{n}$ of $\left\{1, \ldots, m^{3}\right\}$ into $m$-sets can be chosen which satisfies (2.6), e.g., $n=p^{2 r}$ for some prime $p$. Let $Y_{n}:=\operatorname{span}\left\{\psi_{i}\right\}_{1}^{2 n} \cup\{1\}$, where $\psi_{i}(x):=\int_{0}^{x} \delta_{A_{i}}(t) d t, 1<i<2 n$, and $1(t)=1$, with $A_{n+k}:=\{(k-1) m+1, \ldots, k m\}$ for $k=1, \ldots, n$, and $\delta_{A_{1}}$ given by (2.3). Then there exists a linear map $P_{n}: C[0,1] \rightarrow Y_{n}$ so that

$$
\left\|f-P_{n} f\right\|_{\infty}<3\left\|f^{\prime \prime}\right\|_{1} n^{-3 / 2} \text { for all } f \in W_{1}^{2}[0,1] \text {. }
$$

Proof. Set $N:=m^{3}$ and let $S=f(0)+\sum_{0}^{N-1} \alpha_{i}\left(\cdot-x_{i}\right)_{+}$be the broken line of Proposition 1 which agrees with $f$ at its breakpoints $x_{i}=i / N, i=0, \ldots, N$. We define $P_{n} f$ by

$$
P_{n} f:=f(0)+\sum_{i=0}^{N-1} \alpha_{i} \Phi_{i}
$$


with

$$
\Phi_{i}(x):=\int_{0}^{x} \phi_{i}(t) d t,
$$

and $\phi_{i}$ given by (2.4). Then $P_{n}$ is a linear map, and maps into $Y_{n}$ since $\Phi_{i} \in Y_{n}$. Also, by (2.5),

$$
\left\|\left(\cdot-x_{i}\right)_{+}-\Phi_{i}\right\|_{\infty}=\max _{x}\left|\int_{0}^{x}\left[\left(t-x_{i}\right)_{+}^{0}-\phi_{i}(t)\right] d t\right|<m_{i k} / N<N^{-1}
$$

because of (2.6). Thus,

$$
\left\|f-P_{n} f\right\|_{\infty}<\|f-S\|_{\infty}+\left\|S-P_{n} f\right\|_{\infty}<\left\|f^{\prime \prime}\right\|_{1} / N+\sum_{i=0}^{N-1}\left|\alpha_{i}\right| / N<3\left\|f^{\prime \prime}\right\|_{1} N^{-1}
$$

using (1.1) and (1.2), which finishes the proof.

3. The combinatorial problem. A partition $A_{1}, \ldots, A_{n}$ for $\left\{1, \ldots, N=m^{3}\right\}$ consisting of $m$-sets and satisfying (2.6) can be obtained if we can show the following:

(3.1) There exist subsets $C_{1}, \ldots, C_{n}$ of $\left\{1, \ldots, n=m^{2}\right\}$, each having $m$ elements, and such that

(i) no integer is contained in more than $m$ of them and

(ii) for all $i \neq j$, $\operatorname{card}\left(C_{i} \cap C_{j}\right)<1$.

Indeed, if (3.1) is true, then we construct $A_{1}, \ldots, A_{n}$ in that order as follows. With $A_{1}, \ldots, A_{i-1}$ already constructed, $A_{i}$ is to contain, for each $k \in C_{i}$, the lowest integer in $A_{n+k}$ not already contained in some $A_{j}$ with $j<i$, (such an integer exists because of (ii)) and no others. Then $A_{i} \cap A_{j}=\varnothing$ for $i \neq j$ and (i) insures that each $A_{i}$ has exactly $m$ entries. Hence $A_{1}, \ldots, A_{n}$ is a partition for $\left\{1, \ldots, m^{3}\right\}$. Also our construction assures that $\operatorname{card}\left(A_{\nu} \cap A_{n+k}\right)<1$ for $\nu, k$. Further, (ii) insures that, if both $A_{\nu}$ and $A_{\mu}$ for $\nu \neq \mu$ have an entry from $A_{n+j}$, i.e., if both $C_{\mu}$ and $C_{\nu}$ contain $j$, then, for all $k \neq j, A_{n+k}$ cannot contain an entry from both $A_{\nu}$ and $A_{\mu}$.

We now attempt to construct a solution for (3.1) in the form

$$
\left\{C_{1}, \ldots, C_{n}\right\}=\left\{C_{i j}: i=-1, \ldots, m-2, j=1, \ldots, m\right\} \text {, }
$$

with the additional property that, for each $i, C_{i 1}, \ldots, C_{i m}$ is a partition for $\left\{1, \ldots, m^{2}=n\right\}$. This insures (i) and insures (ii) for $C_{i k}$ and $C_{i h}$ with $h \neq k$. In addition, (ii) then requires that

$$
\operatorname{card}\left(C_{i j} \cap C_{h k}\right)=1 \text { for } i \neq h \text { and all } j, k \text {. }
$$

There is, therefore, no loss in generality in choosing

$$
\begin{aligned}
C_{-1, j} & =\{(j-1) m+1, \ldots, j m\}, \quad j=1, \ldots, m, \\
C_{0, j} & =\{j, j+m, \ldots, j+(m-1) m\}, \quad j=1, \ldots, m .
\end{aligned}
$$

If we write the numbers $\left\{1, \ldots, m^{2}=n\right\}$ into a square array $Q$ as follows

$$
Q:=\left(\begin{array}{cccc}
1 & 2 & \cdots & m \\
m+1 & m+2 & \cdots & m+m \\
\cdot & \cdot & \cdots & \cdot \\
m(m-1)+1 & \cdot & \cdots & m \cdot m
\end{array}\right)=:\left(q_{i, j}\right)
$$


then our first partition $C_{-1,1}, \ldots, C_{-1, m}$ consists of the rows of $Q$ and the second partition, $C_{0,1}, \ldots, C_{0, m}$, consists of the columns of $Q$. Consequently, by (3.2), any $C_{i j}$ with $i>0$ must pick exactly one element from each row and from each column of $Q$. This means that we can represent each such $C_{i j}$ as a permutation $\gamma_{i j}$ of degree $m$ with

$$
C_{i j}=\left\{q_{r, \gamma_{i j}(r)}: r=1, \ldots, m\right\} \text {. }
$$

Further, (ii) is insured if $\gamma_{i j}^{-1} \gamma_{h k}$ has at most one fixed point whenever $(i, j) \neq(h, k)$.

With this, we are ready to prove (3.1) in case $m$ is a prime power. In that case, the numbers $1, \ldots, m$ can be identified with the elements of a field (with 1 the multiplicative identity and $m$ the additive identity, say) [6]. Now, for $i=1, \ldots, m$ -1 and $j=1, \ldots, m$, define

$$
\gamma_{i j}(r):=i * r \oplus j, \quad r=1, \ldots, m,
$$

with $*$ and $\oplus$ the field operations. Then $\gamma_{i j}$ is invertible (since $i \neq m$ ), hence a permutation of degree $m$. Further, if $\gamma_{i j}^{-1} \gamma_{i k}(r)=r$, then $j=k$. Hence, $C_{i 1}, \ldots, C_{i m}$ is a partition of $\left\{1, \ldots, m^{2}\right\}$, for all $i$, thus insuring (i). Finally, if $\gamma_{i j}^{-1} \gamma_{h k}(r)=r$ for $r=s$ and $r=t$, then $(i \ominus h) * s=(i \ominus h) * t$, hence either $s=t$, or else $i=h$, but then, as seen just now, also $j=k$. This proves (3.1) for the case that $m$ is a prime power.

We note that we have actually found $m$ more subsets $C_{i}$ than we needed. Also, for $i>0$, the sets $C_{i 1}, \ldots, C_{i m}$ form a Latin square $L_{i}$, and (ii) states that $L_{i}$ and $L_{j}$ are "orthogonal" for $i \neq j$ (see, e.g. [6]). Thus it is known that our problem (3.1) has no solution if $m=6$ (Euler's problem of the 36 officers) and it is unknown already for $m=10$ whether or not (3.1) has a solution.

4. $n$-widths. In this last section, we record various statements about $n$-widths, which follow readily from Theorem 1 by integration and the use of standard arguments from the interpolation of linear operators. Let $d_{n}$ be the $n$-width as given in the introduction and let $\delta_{n}$ be the linear $n$-width as defined by

$$
\delta_{n}\left(U\left(W_{p}^{k}\right)\right)_{q}:=\inf _{L_{n}} \sup _{f \in U\left(W_{p}^{k}\right)}\left\|f-L_{n}(f)\right\|_{q}
$$

where the inf is taken over all linear operators $L_{n}$ whose range has dimension $<n$.

THEOREM 2. For any $n=p^{2 k}, p$ a prime, we have

$$
\delta_{2 n+1}\left(U\left(W_{1}^{2}\right)\right)_{\infty}<d_{2 n+1}\left(U\left(W_{1}^{2}\right)\right)_{\infty}<3 n^{-3 / 2} \text {. }
$$

For any integer $r>2$ and $n>1$,

$$
\delta_{n}\left(U\left(W_{1}^{r}\right)\right)_{\infty}<d_{n}\left(U\left(W_{1}^{r}\right)\right)_{\infty}<\text { const } n^{-r+1 / 2} \text {. }
$$

For any $\alpha>2,1<q<\infty$, and any $n>1$,

$$
\delta_{n}\left(U\left(B_{1}^{\alpha, q}\right)\right)<d_{n}\left(U\left(B_{1}^{\alpha, q}\right)\right)_{\infty}<\text { const } n^{-\alpha+1 / 2},
$$

where $B_{1}^{\alpha, q}$ is the Besov space.

Acknowledgement. We thank Professor A. Pinkus for several valuable comments on our first draft of this paper. 


\section{REFERENCES}

1. C. de Boor and G. Fix, Spline approximation by quasi-interpolants, J. Approx. Theory 7 (1973), 19-45.

2. P. L. Butzer and H. Berens, Semi-groups of operators and approximation, Springer-Verlag, Berlin, 1967.

3. E. Gluskin, On a problem concerning diameters, Soviet Math. Dokd. 15 (1974), 1592-1596.

4. R. S. Ismagilov, Diameters of sets in normed linear spaces, and the approximation of functions by trigonometric polynomials, Russian Math. Surveys 29 (1974), 169-186.

5. B. S. Kashin, The widths of certain finite dimensional sets and classes of smooth functions, Izv. Akad. Nauk SSSR, Ser. Mat. 41 (1977), 334-351. (Russian)

6. H. J. Ryser, Combinatorial mathematics, Carus Monographs, No. 14, 1963.

7. S. B. Stechkin, On the best approximation of given classes of functions by arbitrary polynomials, Usephi Mat. Nauk 9 (1954), no. 1, 133-134.

Mathematics Research Center, University of Wisconsin, Madison, Wisconsin 53706

Department of Mathematics, University of South Carolnna, Columabu, South Carolna 29208

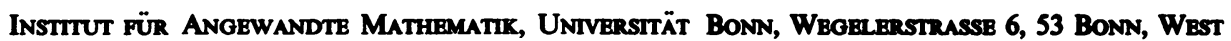
GrRMANY 